\title{
Subject Status Completion Status
}

National Cancer Institute

\section{Source}

National Cancer Institute. Subject Status Completion Status. NCI Thesaurus. Code

C117665.

A term used to describe the state or condition of the completeness of the subject status data. 